\title{
"Alimentar las arcas del Estado": corporaciones agrarias, Estado y política en Córdoba (1995-1999)
}

\author{
["Feeding the State's Coffers": Agrarian Corporations, State, \\ and Politics in Córdoba (1995-1999)]
}

\author{
Gabriel Fernando Carini \\ (Centro de Investigaciones Históricas-Universidad Nacional de Río Cuarto \\ /CIFFyH-UNC/becario CONICET) \\ gcarini@hum.unrc.edu.ar
}

Resumen

El presente artículo tiene como objetivo analizar los procesos de mediación política frente al Estado cordobés de un conjunto significativo de corporaciones agrarias para, en ese marco, dar cuenta de los discursos y representaciones que abonaron no solo una determinada visión sobre el Estado, sino también una crisis de representación de la clase política. En ese contexto, se reactualizaron enunciados a los que históricamente las corporaciones agrarias habían apelado para trazar diagnósticos, exigir demandas y movilizar sus bases sociales. Paralelamente, se tornaron operativos tópicos como el de la reconversión empresaria para explicar la necesidad de que el Estado y los políticos 'modernizaran' sus prácticas. Para alcanzar este objetivo, nos servimos fundamentalmente de la prensa, puesto que la misma constituye un canal habitual de mediatización de la cuestión rural y, por ende, es un espacio privilegiado para el estudio de las representaciones y discursos construidos por estos actores.

Palabras clave: Estado - Corporaciones Agrarias Mediación política - Córdoba

\begin{abstract}
The objective of this article is to analyze the processes of political mediation in front of the Cordoba State of a significant group of agrarian corporations, in order to account for the speeches and representations that supported not only a certain vision about the State but also a crisis of representation of the political class. In this framework, statements to which historically the agrarian corporations had appealed were restated to draw diagnoses, demands and mobilize their social bases. At the same time, topical operatives such as business reconversion became operational to explain the need for the State and politicians to 'modernize' their practices. To this end, we use as source the press mainly, because it constitutes a regular mediation channel for the rural issue, and therefore a privileged space for the study of the representations and discourses constructed by these actors.
\end{abstract}

Keywords: State - Agrarian Corporations Political Mediation - Córdoba

Recibido: 28/05/2017

Evaluación: 23/08/2017

Aceptado: 17/04/2018

Anuario de la Escuela de Historia Virtual - Año 9 - N 14 - 2018: pp. 100-119.

ISSN: 1853-7049

http://revistas.unc.edu.ar/index.php/anuariohistoria 


\section{"Alimentar las arcas del Estado": corporaciones agrarias, Estado y política en Córdoba (1995-1999)}

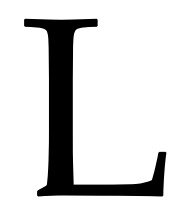

a década de 1990 estuvo marcada por profundas rupturas que supusieron la alteración de la configuración estatal que, desde la primera mitad del siglo XX, había predominado en Argentina. En este sentido, el tránsito hacia una matriz estatal, que conjugó más mercado con menos Estado, ha recibido la atención por parte de los investigadores en ciencias sociales. ${ }^{1}$ Estos trabajos brindaron un panorama sobre las consecuencias del 'retiro' del Estado de áreas sensibles como la educación, la salud y los mercados de trabajo. Asimismo, permitieron comprender el cambio en la dinámica relacional entre los principales actores socio-económicos y el Estado, en particular, a partir de la gravitación de los de carácter transnacional en el diseño de las políticas económicas. ${ }^{2}$ No obstante, quedan todavía por indagar de forma más exhaustiva los procesos de reforma estatal en las realidades provinciales, en particular, en lo que se refiere a la nueva relación fiscal establecida entre el Estado nacional y los Estados provinciales y la necesidad de éstos de proveerse de recursos para atender áreas que hasta ese momento eran responsabilidad del primero. ${ }^{3}$ En este sentido, la cuestión impositiva se constituyó en un problema político que explicó parte de la conflictividad, en especial, con aquellos sectores que tradicionalmente contribuían a sostener el erario público, como los del mundo rural. A pesar de esta cuestión, resulta llamativo que dada la relevancia productiva que revestía (y reviste) la actividad agropecuaria en Córdoba

\footnotetext{
${ }^{1}$ No es nuestro objetivo realizar un estudio pormenorizado de la literatura especializada. No obstante, dentro del amplio abanico de trabajos consideramos significativo mencionar los de Palermo y Novaro (1996), Cavarozzi (1997), Sidicaro (2002), Thwaites Rey (2002), Svampa (2010), Pucciarelli (2011). Una lectura crítica sobre algunos de estos y otros trabajos referidos al periodo menemista puede encontrarse en: Fair (2009). ${ }^{2}$ Desde fines de la década de 1980 se evidenció en las ciencias sociales un creciente interés por explicar las lógicas de acción de los actores empresarios. De forma explícita o implícita estos trabajos abrevaban en lo que se conoce como neocorporativismo. A pesar de no constituir un cuerpo teórico estructurado pueden reconocerse algunas premisas comunes a los planteos de esta perspectiva. En primer lugar, se parte de considerar que las mayores asociaciones de intereses (especialmente las vinculadas con lo económico) desarrollan formas no competitivas de representación, con un cierto grado de obligatoriedad en la participación, contando para ello con el soporte y el apoyo de las instituciones gubernamentales. En segundo lugar, estas asociaciones adquieren cada vez una mayor visibilidad en los procesos de toma de decisiones, no limitándose a influir -de forma más o menos efectiva- en los mismos, sino que su accionar se despliega sobre un amplio abanico de formas de participación directa en la gestión e implementación de las políticas públicas en estrecho contacto con la administración. Por último, en tercer lugar, se afirma que en algunos países se consolidó un sistema de relaciones en el que las mayores asociaciones de intereses económicos actúan monopolísticamente, junto con la participación del Estado, en el establecimiento de formas estables e institucionalizadas para alcanzar acuerdos de cooperación en temas macroeconómicos y en los grandes ámbitos de las políticas redistributivas (Schmitter, 1991; 1995). En esta perspectiva se inscribe nuestro trabajo. Una discusión de la producción sobre corporaciones agrarias la hemos realizado en Carini (2018).

${ }^{3}$ Gerchunoff y Torre (1996) han comentado algunas de las implicancias de este proceso, pero en líneas generales el mismo no se encuentra densamente trabajado.
} 
102 | "Alimentar las arcas del Estado": corporaciones agrarias, Estado y política...

no se hayan indagado las dinámicas políticas generadas en torno al proceso de reforma y los actores corporativos del agro provincial. ${ }^{4}$

Con ese trasfondo, nuestro objetivo es mostrar la mediación política ejercida por un conjunto de corporaciones agrarias de la provincia de Córdoba para propiciar la reducción del impuesto inmobiliario rural (uno de los principales recursos financieros del Estado provincial) para, en ese marco, analizar los discursos esgrimidos por la dirigencia agraria en torno al proceso de ajuste impulsado por el gobierno provincial que dio paso a un creciente cuestionamiento a su representatividad política. ${ }^{5}$ Así, nos detendremos en las representaciones construidas por un sector significativo de la dirigencia agraria de Córdoba en torno a lo político, o sea, sobre todo aquello que constituye a la polis más allá del campo inmediato de la competencia partidaria por el ejercicio del poder (Rosanvallon, 2003, p. 20). Partimos de la hipótesis que, en la lectura del proceso políticoeconómico abierto en 1995 realizada por la dirigencia agraria cordobesa se reactualizaban, por un lado, las representaciones que durante la crisis hiperinflacionaria de 1989 habían legitimado la emergencia de un nuevo régimen social de acumulación y que tenían anclaje en discursos históricamente esgrimidos sobre el Estado y su rol en la economía. Es decir, que ambas situaciones de crisis permitieron la canalización de enunciados, expresiones y frases que remitían a una discursividad de tipo liberal-conservadora caracterizada por una defensa del derecho inalienable de la propiedad privada, la no intervención del Estado en las actividades privadas y una apertura irrestricta de la economía, ${ }^{6}$ lo que habilitaba un espacio de excepción para la implementación de medidas de ajuste. Por otro lado, que la crítica ejercida a la clase política no solo trasuntaba la crisis de representación política que caracterizó el período, sino que volvía operativa la noción de reconversión, tanto para explicar los procesos como para demandar soluciones. Esta idea asimilaba los procesos de profesionalización y empresarialización de las prácticas agronómicas asociadas a los imperativos del agronegocio con la necesidad de que los políticos asumieran conductas más eficientes en la administración de lo político. ${ }^{7}$ En definitiva, consideramos que la utilización de estos múltiples sistemas de representaciones,

\footnotetext{
${ }^{4}$ En esa perspectiva, existen para el caso de la provincia de Córdoba importantes avances no solo sobre el proceso de reforma del Estado provincial, sino también la conflictividad generada y la emergencia de nuevos colectivos que comenzaban a trasladar sus demandas a la esfera pública. Cfr.: Carrizo (2000), La Serna (2001a; 2001b), Ase y Burijovich (2001), Segura (2007), Closa (2010), Gordillo (2010) y Gordillo et al. (2012).

${ }^{5}$ Aquí entendemos por mediación política al conjunto de actos y de actitudes dirigidos a influir de manera más o menos directa y más o menos legal sobre las decisiones quienes tienen el poder en el sistema político o en cada una de las organizaciones políticas, así como en su misma selección, con vistas a conservar o modificar la estructura (y por lo tanto los valores) del sistema de intereses dominante (Pasquino, 1996, p. 180).

${ }^{6}$ Balsa $(2007,2012,2013)$ ha señalado la presencia de tres formaciones discursivas (liberal-conservadora, agrarista y tecnologizante) sobre la base del análisis de los discursos públicos circulantes sobre el sector agropecuario. Cada formación discursiva se caracteriza por la construcción de enunciados, el empleo de ciertos términos y expresiones claves que le otorgan y construyen un sentido determinado sobre la cuestión agraria. Estas formaciones discursivas poseen historicidad y pueden adquirir preponderancia unas frente a otras en determinadas coyunturas históricas.

${ }_{7}^{7}$ Para una caracterización del modelo de agronegocios puede consultarse: Gras y Hernández (2009, 2013).
} 
que impregnaban la visión de los colectivos agrarios sobre el Estado y la cuestión impositiva, fueron recursos que permitieron a la dirigencia agraria cordobesa definir, estructurar y trasladar sus intereses al espacio público y configurar una determinada cultura política.

Nuestro objeto de estudio serán las intervenciones públicas de la dirigencia agraria cordobesa canalizadas a través del diario regional Puntal $(\mathrm{P})$, más precisamente en su sección sobre asuntos agrarios y su suplemento 'Tranquera Abierta' (TA). Esta publicación es de alcance regional, abarcando las ciudades de Río Cuarto y Villa María y se extendiendo hasta la provincia vecina de San Luis. La colección completa de esta publicación se encuentra disponible en la hemeroteca $(\mathrm{H})$ del Archivo Histórico Municipal de Río Cuarto (AHMRC). El análisis de este tipo de fuentes es relevante, puesto que es un medio habitual de mediatización de la ruralidad, así como también un espacio simbólico de reproducción de las representaciones de los sectores agrarios (Carniglia, 2004, p. 288289). Estos soportes constituyen selecciones de datos de la realidad, que se organizan de determinada manera para decir, hacer entender y dar a conocer (Carreras Doallo, 2012, p. 186) y, en consecuencia, son un elemento privilegiado tanto para el estudio de los discursos y representaciones de las entidades como para conocer sus prácticas institucionales. Puntualmente, seleccionaremos los discursos de los dirigentes de la Sociedad Rural de Río Cuarto (SRRC), entidad de primer grado, ${ }^{8}$ y de Confederaciones Rurales de la Tercera Zona (CARTEZ), entidad de segundo grado que reunía a las sociedades rurales de las provincias de San Luis, Catamarca, La Rioja, Mendoza y Córdoba. ${ }^{9}$ Las de esta última provincia históricamente asumieron la conducción de aquellas. Si bien existen otras entidades dentro del andamiaje de representación de intereses agrarios en la provincia de Córdoba -como el caso de las filiales de Federación Agraria Argentina (FAA) y de las organizaciones cooperativas de primer grado nucleadas en la Confederación Intercooperativa Agropecuaria (CONINAGRO)-, la elección se fundamenta en la representatividad que poseían, traducida no solo en la cantidad y relevancia económica de los productores asociados, sino también en la capacidad de mediación política frente al Estado provincial, es decir, en la posibilidad de establecer y/o redefinir las reglas del campo político.

\footnotetext{
${ }^{8}$ Entidad de primer grado nacida en 1938, como efecto del accionar de la Agrupación de Accionistas Ley 11.747. Región Sud de Córdoba, la que entendía que había agotado los fines para los cuales había sido constituida y, por ende, era necesario profundizar la agremiación en otra entidad. La SRRC nucleaba a medianos y grandes productores, especialmente dedicados a la producción agrícola mixta, con un marcado predominio de la ganadera, así como de actividades vinculadas a la genética animal en cabañas. En cuanto al perfil social de sus asociados, la institución agrupaba mayoritariamente a profesionales (especialmente abogados, ingenieros agrónomos y médicos veterinarios), que poseían cierta diversificación de sus actividades (en muchos casos asociadas con la venta de servicios y productos agrícolas) y estaban ligados al poder político, especialmente al provincial y nacional (Carini, 2011, 2015, 2017).

${ }^{9}$ Entidad de segundo grado nacida en junio de 1940 por iniciativa de la SRRC para mejorar el posicionamiento de sus bases sociales frente a la comercialización de carne vacuna. CARTEZ ingresó en 1947 a Confederaciones Rurales Argentinas. De esta forma, la dirigencia cordobesa contó con un mecanismo asociativo para trasladar sus demandas al ámbito nacional (Carini, 2011, 2015, 2017).
} 
104 | "Alimentar las arcas del Estado": corporaciones agrarias, Estado y política...

El presente artículo se organiza en tres apartados. El primero tiene por objetivo mostrar las características que históricamente asumieron algunos de los rasgos de los perfiles institucionales de las entidades mencionadas, en especial, los discursos y las representaciones. Esto nos permite observar las continuidades y rupturas experimentadas en estas dimensiones en la coyuntura estudiada. En el segundo apartado se reconstruyen los discursos y representaciones realizados por la dirigencia agraria cordobesa en torno al Estado entre 1995 y 1999. Finalmente, el tercer apartado está destinado a dar cuenta de algunos aspectos que asumió la crisis de representación política durante el período considerado.

\section{Estado, política y cuestión impositiva: discursos y representaciones históricas de las corporaciones agrarias cordobesas}

Las políticas agropecuarias -y especialmente las de carácter redistributivo, es decir, las que suponen transferencias de recursos tanto al interior del sector agropecuario como entre este y otros sectores económicos (Martínez Nogueira, 1988)- generan las mayores críticas de las corporaciones agropecuarias. Constituyen, al mismo tiempo, un punto de convergencia (especialmente en los aspectos relativos a los índices de precios y tipo de cambio) entre las asociaciones del agro, puesto que, a partir del diagnóstico y análisis de sus implicancias, se estructuran acciones de fuerza, incluso, por parte de aquellas entidades que sostienen principios o valores ideológicos diametralmente opuestos entre sí (Martínez Nogueira, 1988; Lattuada, 1992; Makler, 2007; Sanz Cerbino, 2014; Carini, 2017).

Como contracara, la política impositiva genera fisuras en la red de representación de los intereses rurales. Aunque existe acuerdo en cuestionar la 'presión tributaria' ejercida por el Estado, el mismo se desvanece cuando se analiza cómo se distribuye la transferencia de recursos -vía la política impositiva- que se opera sobre la base de los precios y de los impuestos a la exportación. Así, el conflicto intersectorial es internalizado y se convierte en cuestión intrasectorial (Martínez Nogueira, 1988, p. 316; Lattuada, 1992).

Respecto a estas cuestiones, la dirigencia agropecuaria de Córdoba construyó históricamente una crítica vertebrada sobre tres aspectos. En primer lugar, se puede observar en las interpelaciones públicas de los directivos de la SRRC y de CARTEZ un cuestionamiento general al sistema tributario. Se desaprobaba tanto la cantidad de tasas y tributos que gravaban la actividad agropecuaria como su superposición. Especialmente, observaban la falta de coordinación entre los diferentes niveles estatales y sostenían la necesidad de una simplificación en las prácticas tributarias. La conjunción de estos factores derivó en el diagnóstico de que el Estado ejercía sobre el sector una fuerte 'presión fiscal' 
que tenía por efecto inhibir la producción y dejar al margen de la supervivencia al productor, situación que evidenciaba la ausencia de una clara 'política agropecuaria'. ${ }^{10}$

En segundo lugar, estuvo presente en la mediatización de las demandas de la dirigencia agraria un constante rechazo a los impuestos que pagaban los derechos de exportación, las denominadas 'retenciones', a los réditos personales o a las ganancias y al impuesto a los consumos (IVA). En líneas generales, se entendía que estas cargas eran 'excesivas' y 'discriminatorias' y se buscaba su reducción o directamente su eliminación, puesto que constituían una acción de 'rapiña' del Estado, destinada a financiar sus déficits. Los períodos en los que se anuló o redujo drásticamente este tipo de gravámenes, en especial las retenciones, fueron 'aplaudidos' y considerados una 'justa reivindicación'.11

Por último, y, en tercer lugar, el accionar de la SRRC y de CARTEZ se dirigió a buscar morigerar la incidencia del Impuesto Inmobiliario Rural en los costos de las explotaciones agropecuarias de sus asociados. Esta cuestión generó permanentes contrapuntos con el Estado cordobés. De forma similar a lo anteriormente enunciado, los argumentos apuntaban a mostrar lo gravoso de este tipo de impuesto, la arbitrariedad del Estado y su incapacidad para administrar correctamente los recursos provinciales. Se adicionaba, además, un cuestionamiento a la progresividad del Inmobiliario Rural. ${ }^{12}$ Esta contribución representaba uno de los principales ingresos de la renta pública de la provincia que -con diferentes variantes- se encontraba vigente desde las primeras décadas del siglo $\mathrm{XX}$ y fue foco de pujas y tensiones entre el Estado provincial, los comerciantes de la ciudad de Córdoba y los propietarios rurales. ${ }^{13}$

Al mismo tiempo, se puede observar que estas críticas estructuraron una determinada representación del Estado, en la que históricamente el mismo aparecía como un agente que, con 'reglamentaciones excesivas' y 'perniciosas', atentaba contra la libre empresa, la libertad de comercio y la propiedad privada. ${ }^{14}$ En este sentido, se consideraba que su acción debía limitarse a:

\footnotetext{
${ }^{10}$ Cfr. AHMRC, H, EP, domingo 04/09/1956, p. 4; AHMRC, H, EP, domingo 06/09/1964, p. 4; AHMRC, H, $E P$, domingo 08/09/1974, p. 2.

11 AHMRC, H, EP, domingo 02/09/1961, p. 9; AHMRC, H, EP, domingo 07/09/1969, p. 4; AHMRC, H, EP, domingo 05/09/1982, p. 7.

${ }^{12}$ AHMRC, H, EP, domingo 05/09/1959, p. 4; AHMRC, H, EP, domingo 04/09/1960, p. 4; AHMRC, H, EP, domingo 1966, p. 2; AHMRC, H, EP domingo 02/09/1973, p. 4; AHMRC, H, EP, domingo 08/09/1974, p. 4; AHMRC, H, LC, domingo 07/09/1986, p. 16.

${ }^{13}$ Con dificultades, desde fines del siglo XIX, el Estado provincial procuró gravar la propiedad inmueble. A partir de 1925, con el gobernador Ramón J. Cárcano, se modificó la política fiscal con cambios en las avaluaciones de los predios y se impuso la fijación de un impuesto por hectárea con un doble objetivo: financiar proyectos de obra pública y desestimular el latifundio, lo que permitía afianzar la pequeña propiedad. Con Amadeo Sabattini (1936-1940) se diseñó una política fiscal que instrumentó un impuesto progresivo sobre la propiedad de la tierra y un gravamen adicional al ausentismo de los dueños. Ambos tendían a crear condiciones pensadas como medios de presión a los grandes propietarios y para obligarlos a mermar su patrimonio inmobiliario con el fin de disminuir la carga impositiva. A estas medidas se agregaba una reforma al impuesto a las grandes herencias, que buscaba inducir a la subdivisión de extensos dominios (Cfr. Converso, 2008; Tcach, 2006).

${ }^{14}$ AHMRC, H, EP, domingo 02/09/1961, p. 9.
} 
106 | "Alimentar las arcas del Estado": corporaciones agrarias, Estado y política...

“(...) una tarea de planificación en los grandes problemas económicos y financieros del país; es uno de sus deberes ineludibles e indelegables, pero esta planificación no debe ir más allá de lo que este término estrictamente significa. La ejecución debe quedar a cargo de la actividad privada, a quien el gobierno debe tratar de crear las mejoras condiciones para su desenvolvimiento. (...) para que se incremente la producción pecuaria, será necesario que el gobierno nacional o provincial con sus leyes y decretos y en general con todo el régimen legal, posibilite a la actividad privada su desarrollo e incremento, y no que el Estado trate de tomarlo a su cargo compulsivamente, mediante expropiaciones y creación de Institutos o reparticiones, de lo que resulta una mayor burocracia y en definitiva un menor rendimiento, comprobado y corroborado por experiencias propias y extrañas." 15

Aquí nos interesa resaltar la defensa que la dirigencia, casi de forma invariable, realizaba de la no intervención del Estado en la actividad agropecuaria. Asociado a esta cuestión, se abogaba por un Estado que eliminara la excesiva burocratización y que redujera su acción al mínimo; fundamentalmente, que no ampliara desmesuradamente el gasto público que -en el discurso de la dirigencia agraria- era financiado exclusivamente por las divisas que generaba el agro. Se pretendía, en definitiva, un Estado 'racional' y 'eficiente'16:

“Necesitamos la quita de las retenciones y la creación de un sistema impositivo justo y sencillo que no sea discriminatorio y esté sustentado por bases realistas de recaudación a fin de adecuar a las mismas los gastos del Estado (...) Cuando reclamamos la quita de retenciones la gente debe entender que no es algo caprichoso, antojadizo de nuestra parte, desconociendo las necesidades que tiene el Estado de juntarse con fondos para solucionar algunos problemas realmente graves que tiene el país. Lo que pasa es que este año, que se exportaron entre 4 y 5 mil millones de dólares del sector, con retenciones del orden del 25 al 27 por ciento que representan 1.182 millones de dólares de ingresos para el Estado, ingresos que son seguros porque no hay posibilidades de evadirlos y entonces el gobierno echa mano de ellos porque es un método rápido y eficiente de hacerse de fondo".$^{17}$

Teniendo en cuenta lo enunciado, podemos afirmar que la discursividad generada por la dirigencia agraria de la SRRC y de CARTEZ puede identificarse por el predominio de una matriz de tipo liberal-conservadora (Balsa, 2007; 2012; 2013). ${ }^{18}$ De esta forma, su discurso se construyó históricamente a partir de un componente marcadamente antiestatista. La crítica al carácter 'desmedido' y 'discriminatorio' de los impuestos que recaían sobre el sector agropecuario constituía la simiente para la construcción de una vi-

${ }^{15}$ AHMRC, H, EP, domingo 02/09/1959, p. 4-5.

16 Ver, por ejemplo: AHMRC, H, P, sábado 01/09/1990, p. 4. AHMRC, H, P, domingo 01/09/1991, p. 24. AHMRC, H, P, sábado 05/09/1992, p. 3 y ARHMRC, H, SA, viernes 05/09/1993, p. 34.

${ }_{17}$ AHMRC, H, P, sábado 01/09/1990, p. 4. El destacado es nuestro.

${ }_{18}$ Para profundizar en esta cuestión se puede consultar: Carini (2015). 
sión del Estado como un actor 'ineficiente' e 'incapaz' de resolver las situaciones de crisis por las que atravesaba el 'campo'. La solución de este problema requería de una imperiosa reducción del 'gasto público', la 'racionalización' de la administración pública y, fundamentalmente, de la no 'injerencia' del Estado en los 'negocios privados'. ${ }^{19}$ Estas concepciones generales que describimos sobre el Estado y la cuestión impositiva tuvieron profundas implicancias durante el período analizado tanto en el plano simbólico como en el político. Estas representaciones históricas se re-actualizaron y cobraron centralidad en la dinámica política de la década de 1990, contribuyendo a la legitimación de medidas tendientes a la reducción del Estado y de apertura de los mercados. Igualmente, abonaron también los consensos que sostuvieron el nuevo régimen social de acumulación y se erigieron como límites a la acción colectiva de las entidades.

\section{2. "(...) que el Estado aporte su propia cuota de sacrificio":20 representaciones y tensiones en torno al Estado cordobés}

Históricamente, los sujetos agrarios nucleados en la SRRC y, por su intermedio, en CARTEZ implementaron diversas estrategias de mediación política frente a los gobiernos provinciales a los fines de que se les morigeraran las cargas impositivas. Estas acciones encontraban funcionarios más o menos permeables a las demandas, especialmente cuando se dirigían a solicitar la prórroga de las cuotas del Inmobiliario Rural o buscaban la declaración de emergencia agropecuaria que tenía un efecto similar, dependiendo de cada caso, sobre las cargas provinciales. Asimismo, la SRRC era asiduamente consultada por la repartición provincial del área agropecuaria para la estructuración de planes destinados al sector. En líneas generales, existía una relación cordial que se traducía, entre otras cosas, en una participación activa de las autoridades provinciales en las ferias anuales organizadas por la entidad. Cabe recordar que un número significativo de socios desempeñaba funciones en diferentes dependencias del gobierno o bien tenía acceso a los funcionarios, dado que compartían los mismos espacios de sociabilidad política o privada. ${ }^{21}$ Desde mediados de la década del ‘90 se produjo un quiebre en esta dinámica y se abrió un período de creciente confrontación.

La salida anticipada del gobierno de Eduardo Angeloz y la asunción de su par radical Ramón Mestre fue la expresión no solo de una crisis política, sino también del deterioro social y económico en el que se encontraba sumergida la provincia, lo que abonó la conflictividad social (Closa, 2010; Arriaga y otras, 2012). Desde la columna institucional de la SRRC se realizaba un análisis de la realidad provincial y se solicitaba a los funcionarios públicos la celeridad en decisiones que recompusieran la situación. Este diagnóstico, como otros realizados por la entidad, se inscribía en una red conflictual previa, que ca-

${ }_{19}$ Una caracterización histórica de las dimensiones de esta discursividad puede encontrarse en Carini (2017a).

${ }^{20}$ AHMRC, H, P, 15/09/1996, p. 5. Declaraciones de Víctor Tonello, presidente de la SRRC.

${ }^{21}$ Algunos elementos que permiten observar estos vínculos los hemos reconstruido en: Carini (2017).

Anuario de la Escuela de Historia Virtual - Año 9 - N 14 - 2018: pp. 100-119. ISSN 1853-7049 
108 | "Alimentar las arcas del Estado": corporaciones agrarias, Estado y política...

racterizaba su práctica de mediación política ejercida sobre el Estado provincial. Se encontraba, además, tamizado por representaciones históricas inscriptas en una matriz liberal-conservadora que encontraba en las prácticas desarrolladas por el Estado el origen de todas las crisis y que había servido para estructurar un consenso en torno a las políticas neoliberales del gobierno de Menem (Carini, 2014a). En ese contexto, se tornó operativo un nuevo argumento, que estaba relacionado con la necesidad de que el Estado llevara adelante una 'reconversión' similar a la que estaba atravesando el sector privado:

"La afligente situación de los empleados públicos, jubilados y proveedores del Estado de la Provincia de Córdoba, habida cuenta del pernicioso atraso de sueldos y pagos por prestación de servicios y suministros de insumos, ha creado ya un cuadro socio-económico de alta tensión, que perturba la vida comunitaria, propiciando un ambiente cargado de acechanzas. (...) Lo que nos preocupa es la falta de soluciones concretas para un problema que ha alcanzado ya un nivel de gravedad extremadamente dañino para la normal convivencia. (...) a los productores se nos exige eficiencia y reconversión empresaria. Parece que esta definición no incluye a la administración pública, ya que cualquier análisis que pudiéramos hacer, en el orden provincial o nacional, nos llevaría a la misma conclusión de un desorden generalizado, cuando no a un despilfarro inaceptable. Así las cosas, no habrá ingresos, por más importantes que ellos sean, que soporten semejante carga de irregularidades y 'desprolijidades'. La situación, evidentemente, ha escapado a los cánones tradicionales" ${ }^{22}$

Entonces, ante el desafío de ordenar la economía y sosegar los niveles de conflictividad emergente, el nuevo gobernador respondió con la implementación de un programa de medidas tendientes a la disminución de gastos y a la 'racionalización' en el funcionamiento del Estado, sustentado en la Ley de Emergencia sancionada en los meses previos (Closa, 2010, pp. 482-483). Básicamente, las medidas apuntaron a una abrupta reducción del tamaño del Estado provincial y al intento -en ocasiones exitoso- de privatización de empresas estatales. Así, por ejemplo, se dispuso una reducción del 30\% de los sueldos de la Administración Pública y de cinco a tres días de trabajo, salvo para los trabajadores de la educación y la policía. Se eliminaron ministerios: de once carteras quedaron cinco y dejó de funcionar el 50\% de las subsecretarías; se sustituyeron los directorios de la Dirección Provincial de Aguas y Saneamiento (DIPAS), la Empresa Provincial de Energía de Córdoba (EPEC) y Vialidad y Arquitectura por un solo funcionario o interventor; se rebajó, además, la coparticipación a los municipios en un orden del 9\% (Closa, 2010, pp. 483-484). ${ }^{23}$

En ese proceso también se concebía la necesidad de incrementar el nivel de recaudación y, en tal sentido, se estudió la posibilidad de imponer nuevas cargas fiscales. Fue este aspecto el que generó uno de los principales contrapuntos entre las corporaciones

\footnotetext{
${ }^{22}$ AHMRC, H, TA, viernes 07/07/1995, p. 7.

${ }^{23}$ Los conflictos que nacieron de este proceso fueron integralmente abordados en Gordillo (2010) y Gordillo et al. (2012) a donde remitimos.
}

Anuario de la Escuela de Historia Virtual - Año 9 - N 14 - 2018: pp. 100-119. ISSN 1853-7049 
agropecuarias y el gobernador Mestre. Esta posibilidad reactualizó los argumentos que contraponían eficiencia del sector productivo versus ineficiencia del Estado:

\begin{abstract}
"¿No es una verdadera injusticia que siempre sean castigados, con más impuestos, los que cumplen debidamente con sus obligaciones? ¿Nos es acaso ineficiente esta inoperancia del Estado frente a situaciones irregulares? Y habría otros tantos interrogantes, que incluso provocan dudas sobre la necesaria transparencia de las cuentas públicas. Todo esto causa irritación y provoca rebeldía. Si la ley es pareja, pagamos todos. Si son muchos los que no pagan lo debido, es que existe ineficiencia, descontrol. (...) Por último decimos que en esto, como en todas las actividades, el ejemplo debe partir de arriba ¡Qué nadie quede excluido de pagar sus impuestos, sean altos funcionarios, legisladores, etc.! (...) se insiste con que debemos ser competitivos. Producir mejor, para obtener mejores rendimientos. El sector agropecuario, a costa de grandes sacrificios, lo está haciendo. Pero, para poder competir, es necesario contar con una sana política de costos. Ya se sabe que los impuestos se constituyen en el índice de mayor incidencia de nuestros costos de producción". ${ }^{24}$
\end{abstract}

Estas cuestiones, lejos de saldarse, se agudizaron y constituyeron uno de los elementos que tensionaron las relaciones entre las entidades agropecuarias y la gestión de Mestre. En ese marco, los clásicos reclamos sobre el sistema de valuación de los campos y el inmobiliario también ocuparon un espacio central en las críticas de las entidades. Los argumentos cuestionaban la progresividad del impuesto y lo presentaban como una injusticia que padecía el sector y como una acción de rapiña que el Estado ejercía sobre el mismo. Se apelaba de esta forma a argumentaciones que tradicionalmente componían el repertorio discursivo de la SRRC, reactualizando la imagen de un Estado ineficiente.

\begin{abstract}
"Las empresas privadas, por su propia supervivencia, están aprendiendo a racionalizar sus cuentas. Las que aún no lo han hecho, deberán hacerlo cuanto antes, ya que las condiciones económicas son cada vez más severas. Frente a esta actualización de la actividad privada, entendemos que el Estado aún no ha hecho la parte que le corresponde. Así se produce un desfase negativo entre los ingresos genuinos y los gastos, lo que trae, como consecuencia, la necesidad de acudir a una mayor imposición fiscal, que reciente el normal desenvolvimiento de todos los sectores de la sociedad. Frente a la desesperación de los balances que 'no cierran', el Estado provincial, vuelve cada vez más a acudir a mayores cargas fiscales, sin advertir, pareciera, que los distintos sectores económicos ya no admiten tales aumentos." 25
\end{abstract}

Otro contrapunto significativo, que se sumó a la cuestión impositiva, fue el del ajuste propiciado desde el Ejecutivo provincial en el área de educación y que implicó la disminución de partidas presupuestarias y, en algunos casos, el cierre de escuelas rurales e institutos agro-técnicos. Sin embargo, esta problemática, aunque despertó malestar y fue señalada en varias oportunidades por la dirigencia agraria, no cobró la dimensión que

${ }^{24}$ AHMRC, H, TA, viernes 20/10/1995, p. 7. Se puede consultar en el mismo sentido: AHMRC, H, TA, viernes 17/11/1995, p. 7 y AHMRC, H, TA, viernes 15/12/1995, p. 7.

${ }_{25}$ AHMRC, H, TA, viernes 09/06/1995, p. 7.

Anuario de la Escuela de Historia Virtual - Año 9 - N 14 - 2018: pp. 100-119. ISSN 1853-7049 
110 | "Alimentar las arcas del Estado": corporaciones agrarias, Estado y política...

sí adquirió la cuestión impositiva y el accionar del Estado asociado a ella. Los argumentos de los dirigentes de la SRRC apuntaban a resaltar la función de las escuelas rurales para evitar el desarraigo y el éxodo de la población rural. Además, se sostenía que estas escuelas eran una 'inversión' y no un 'gasto'. ${ }^{26}$

A pesar de lo anterior, en la red conflictual se remitía fundamentalmente a dos cuestiones: por un lado, los sucesivos pedidos de apertura de líneas de crédito para financiar las campañas agrícolas y de refinanciación de las deudas con el Banco Provincia y Social. En estos reclamos se hacía explícita, además, la preocupación por la situación financiera de los productores ante una posible privatización de las entidades bancarias. ${ }^{27}$ Por otro lado, la cuestión fiscal fue expresada en la solicitud de implementación de la emergencia agropecuaria provincial, la cual canalizó el principal descontento de los productores agropecuarios cordobeses.

Desde fines del año 1996 las distintas entidades agropecuarias que operaban en el espacio provincial habían solicitado y mantenido reuniones tanto con legisladores y representantes de la cartera del sector como con el propio gobernador con el fin de expresar la necesidad de declarar la emergencia agropecuaria provincial. Así, por ejemplo, la SRRC se entrevistó primero con la Comisión de Agricultura y Ganadería de la Cámara de Diputados de la provincia y, posteriormente, accedió a una reunión con Mestre. En estas instancias se sostenía que “(...) habida cuenta del importantísimo aporte que realiza el sector agropecuario al erario público, lo que se requiere es una mínima ayuda, traducida en la prórroga de vencimientos crediticios". ${ }^{28}$ Las contramarchas y evasivas del gobernador para dar cabida a los reclamos de la entidad determinaron la posibilidad de intimar judicialmente para que se sancionara la emergencia. El vicepresidente de CARTEZ, y vocal de la SRRC, explicaba que la negativa del Estado provincial se había transformado en "un nuevo cuento" y que el gobernador "ya no tiene voluntad política de aplicarla" y agregaba que: "Sería muy torpe no blanquear esta situación puesto que la emergencia no significa que se perdone el pago sino que simplemente se da un tiempo más para que se pueda cumplir" ${ }^{29}$

Finalmente, la emergencia fue declarada hacia fines de abril del año 1997 pero se consideró insuficiente, puesto que, al haberse aplazado la firma del decreto, muchos productores habían entrado ya en mora con el sistema financiero provincial y nacional. ${ }^{30}$ Esta cuestión permaneció en un estado de latencia y se reactivó al promediar el año con pedidos de extensión del plazo de excepción señalado por la emergencia agropecuaria. En un pedido conjunto de CARTEZ, FAA y CONINAGRO le solicitaban al gobernador:

\footnotetext{
${ }^{26}$ Algunas referencias sobre este tópico las podemos encontrar en: AHMRC, H, $P$, martes 10/09/1995, p. 14; AHMRC, H, P, domingo 15/09/1996, p. 4 y 5.

${ }^{27}$ Cabe advertir que estas demandas eran esgrimidas, principalmente, por FAA y, en menor medida, por CARTEZ. Ver, por ejemplo: AHMRC, H, P, viernes 22/03/1996, p. 3; AHMRC, H, SA, sábado 06/04/1996, p. 7; AHMRC, H, SA, viernes 10/05/1996, p. 2.

${ }_{28}$ AHMRC, H, SA, viernes 13/12/1996, p. 3 y AHMRC, H, SA, viernes 20/12/1996, p. 3.

${ }^{29}$ AHMRC, H, $P$, jueves 20/03/1997, p. 24.

30 AHMRC, H, P, sábado 26/04/1997, p. 24.
} 
“1) Continuar receptando las solicitudes de emergencia agropecuaria para todos los productores de la provincia en esa situación, 2) Extender el plazo de la emergencia, previsto originalmente hasta el 31 de agosto del presente año, a una fecha posterior a determinar por la autoridad provincial, 3) Instrumentar un plan de regularización de deudas impositivas que se canalizará a través de las entidades agropecuarias. Este plan contempla plazos más extensos que los que actualmente emplea DGR y a un interés mensual del 1\%,4) Analizar la posibilidad de otorgar, por parte del Banco Social, créditos personales a productores en emergencia, 5) Diferenciar los casos puntuales de productores en emergencia de aquellos en situación de desastre agropecuario, considerándolas como dos situaciones distintas según lo establece la ley". ${ }^{31}$

La situación no se saldó y se asistió a un creciente clima de conflicto y descontento que, sin llegar a medidas de acción directa por parte de la SRRC o CARTEZ, ${ }^{32}$ se expresó en las intervenciones públicas de los dirigentes rurales. ${ }^{33}$ Mestre logró descomprimir la protesta rural al asumir una actitud más contemporizadora. Para ese momento, se había logrado sanear parte de las cuentas públicas, lo que le permitía al gobierno un mayor margen de acción para lograr acuerdos con los actores socio-económicos (Closa, 2010). En ese sentido, se abrieron canales constantes de diálogo con las entidades agrarias que les permitieron expresar sus reclamos, vinculados especialmente con la situación de los productores del sur cordobés afectados por las inundaciones, la implementación de planes de regularización de deudas por mora en el Impuesto Inmobiliario Rural y la apertura de líneas de créditos del Banco Córdoba. También consideraban auspicioso el compromiso asumido por el gobierno de volver a poner en funcionamiento el Consejo Asesor Agropecuario que tenía como función analizar, evaluar y atender las dificultades que fueron surgiendo de cada uno de los temas como: caminos rurales, Impuesto a los Ingresos Brutos, escuelas rurales, conservación de los suelos, etc. ${ }^{34}$

A pesar de esta flexibilización en la postura del titular del Ejecutivo provincial, al finalizar su mandato, los dirigentes rurales remarcaron que "Mestre se acordó tarde de escuchar al campo". ${ }^{35}$ De esta forma, al clima de deterioro económico de los productores cordobeses se sumó el estilo político rígido del gobernador, confrontativo, renuente a

${ }^{31}$ AHMRC, H, SA, viernes 13/06/1997, p. 2.

${ }^{32}$ A diferencia de las citadas entidades, la FAA realizó una movilización frente a la Casa de las Tejas que tuvo un fuerte componente simbólico al visibilizar, mediante los tractores estacionados frente al palacio de gobierno, la protesta de un conjunto significativo de productores rurales en un espacio eminentemente urbano. Entre los factores que determinaron el 'tractorazo' se encontraban la dilación del Gobernador en declarar la emergencia agropecuaria, los restringidos alcances de la misma y la ausencia de créditos para paliar la situación. Cfr. AHMRC, H, P, martes 15/07/1997, p. 24; AHMRC, H, P, 16/07/97, p. 24; AHMRC, H, $P$, jueves 17/07/97, pp. 20-21; AHMRC, H, SA, viernes 18/07/1997, pp. 2-3. Esta cuestión se debió, tal vez, a la heterogeneidad de la base social de la entidad y que fue, justamente, la más golpeada por los procesos de diferenciación social en el agro. Ver: Carini (2017).

${ }^{33}$ Cfr. AHMRC, H, SA, viernes 31/07/1998, p. 16; AHMRC, H, SA, viernes 29/01/1999, p. 3; AHMRC, H, $S A$, viernes 15/08/1998, p. 16; AHMRC, H, $P$, jueves 11/03/1999, p. 12; AHMRC, H, P, miércoles 10/03/1999, p. 13 y AHMRC, H, $S A$, viernes 12/03/1999, p. 4.

${ }_{34}$ AHMRC, H, TA, viernes 21/08/1998, p. 15.

35 AHMRC, H, P, sábado 13/03/1999, p. 22. 
112 | "Alimentar las arcas del Estado": corporaciones agrarias, Estado y política...

persuadir y a encontrar apoyos políticos para su gestión (Riorda, 2004; Closa, 2010, p. 486). Esta cuestión amplificó el conflicto y, además, fue una expresión cabal del cuestionamiento a los dirigentes y partidos políticos configurado hacia mediados de la década.

\section{3. "En esta provincia castigamos con nuestro voto a un gobernador soberbio y prepo- tente": ${ }^{36}$ una lectura de la crisis de representación política en clave provincial}

Durante la década de 1990 se manifestó de manera más evidente una crisis de las capacidades estales en combinación con una aguda percepción sobre la capacidad de los partidos políticos en general, y de la dirigencia política en particular, para dar solución a los problemas de la ciudadanía, especialmente a los de índole económica. ${ }^{37} \mathrm{~A}$ este marco se le adicionó en la provincia de Córdoba, durante el gobierno de Ramón Mestre, una modalidad de gestión de la política reacia a forjar canales fluidos de diálogo con los diferentes actores de la sociedad civil cordobesa y que se caracterizó, desde lo comunicacional, por un estilo centrado exclusivamente en lo inventarial, cual comunicación contable, esencialmente de carácter retrospectivo, lo que marcaba un abrupto corte con respecto a la gestión de Eduardo Angeloz (Riorda, 2004, pp. 132-133).

Probablemente, fueron las circunstancias coyunturales las que produjeron dicha variación en Mestre, puesto que no solo asumió en forma anticipada, sino que debió afrontar la delicada situación económica por la que atravesaban las arcas provinciales. Determinó un tipo de estrategia política que, aunque en el corto plazo contribuyó a ensanchar la distancia entre la sociedad y sus representantes, fue favorablemente receptada por la dirigencia ruralista del sur de Córdoba. El presidente de CARTEZ y ex presidente de la SRRC trazaba la herencia recogida, cuando señalaba que "Mestre no recibió un gobierno sino una brasa ardiente" y fue ese "estado de calamitoso" el que le obligó a tomar medidas "de extrema dureza". El dirigente, a la par de que manifestaba su "indignación por encontrar la provincia prácticamente vaciada", reclamaba una investigación y que se castigara a los responsables, sentenciando: "Mucha tierra se ha juntado en la casa en doce años. Barra y limpie, que para todo eso cuenta con todo el apoyo del ruralismo". ${ }^{38}$ Así, estas intervenciones públicas de la dirigencia ruralista traían nuevamente como parámetro para diagnosticar e instrumentar soluciones la idea de que la 'política' debía 'reconvertirse', tal como había acontecido con la producción:

\footnotetext{
“(...) los partidos políticos son responsables de mantener y preservar una democracia que costó mucho conseguir y deben ser ejemplo de cordura y cultura cívica ante la sociedad (...) los argentinos sentimos una urgente necesidad de gobernantes dignos de nuestro respeto y de nuestro aprecio. Y le pedimos a los partidos políticos
}

\footnotetext{
${ }^{36}$ SRRC, comunicado de prensa, 17/03/1999.

${ }^{37}$ Algunas de las dimensiones implicadas en este proceso fueron trabajadas en Carini (2014).

${ }^{38}$ AHMRC, H, P, sábado 10/09/1995, p. 14.
} 
que se reconviertan, así como se pedía a los productores y a todos los sectores que lo hicieran". ${ }^{39}$

En esa misma línea discursiva, el presidente de la SRRC también encaraba un espinoso diagnóstico de la situación de la provincia y reclamaba "penas y sanciones para aquellos funcionarios que no cumplan con su deber y con su trabajo, para el que cobran y mucho" y no solo señalaba al ex gobernador Angeloz como responsable de la crisis sino que cuestionaba su actitud de asumir como senador nacional "(...) Nos duele que [aquellos que] son responsables de esta grave crisis se consideren todavía con títulos suficientes como para aspirar a nuevas funciones". Asimismo, planteaba varios reclamos vinculados a la falta de modernización tanto del sistema político como de los partidos que derivó en una restricción de las posibilidades de mediación política y de participación institucional. Entre otros aspectos, se solicitaba la eliminación de las listas sábana en las elecciones, la independencia de poderes, una acción más dinámica de los órganos de contralor y una fuerte reducción del gasto público. Y en ese marco, retomaba la necesidad de que el sector político se reconvirtiera:

“El pueblo requiere señales de que el ajuste es igual para todos, porque molesta ver cómo se malgastan los recursos que aportamos, en tanto que los servicios esenciales, como la salud, la educación y la seguridad dejan mucho que pensar (...) hagan de la política una forma de vida y no un medio de vida". ${ }^{40}$

De esta forma, la actitud en principio contemplativa del ruralismo cordobés respecto a la situación heredada por Mestre ya canalizaba una crítica a determinadas prácticas políticas que se agudizó conforme avanzó el proceso de ajuste iniciado por el nuevo gobernador. La necesidad de recuperar el orden para superar la cesación de pagos y la quiebra de las cuentas públicas fueron los argumentos que le llevaron a plantear un modelo de Estado, pero, además, y consecuente con ello, un modelo de comunicación. Mestre mostró una constante en campaña y en su gobierno: discursos duros, rostro adusto, voz enérgica y mensajes despojados de retórica persuasiva partidaria (Riorda, 2004, p. 132). La dirigencia rural continuaba apelando a un discurso que acentuaba el carácter sobredimensionado del Estado y, por ende, la necesidad de reducirlo, aunque en la coyuntura de creciente cuestionamiento a la clase política se incorporaban también tópicos sobre la pérdida de capacidad para interpelar las necesidades de la sociedad civil. En este sentido, se planteaba desde el palco de la SRRC:

“(...) reducir el gasto que ha seguido creciendo en forma ininterrumpida para manejar la cosa pública castigando los casos de corrupción y para controlar la evasión

\footnotetext{
${ }^{39}$ AHMRC, H, $P$, sábado 10/09/1995, p. 14.

${ }^{40}$ AHMRC, H, P, miércoles 11/09/1996, p. 2. 
114 | "Alimentar las arcas del Estado": corporaciones agrarias, Estado y política...

fiscal (...) Proponemos dar ejemplos de austeridad por parte de los funcionarios ya que el pueblo necesita señales claras de que el ajuste lo tenemos que hacer todos, sin excepción". ${ }^{41}$

Para el año 1999, una vez superada la etapa de ajuste más severo y cuando se percibía cierta normalización en las cuentas públicas del gobierno de la provincia (Closa, 2010), el quiebre del lazo de confianza y de identificación que unía a los partidos políticos con sus representados parecía irreversible. A similitud de lo que acontecía a nivel nacional con el denominado 'voto bronca', en el ámbito de la provincia la dirigencia ruralista apelaba a la implementación de 'urnazos' como muestra del descontento frente a "(...) las autoridades que continúan haciendo oídos sordos a los reclamos". ${ }^{42}$ Bajo esta modalidad se interpelaba a la sociedad civil en general y a las bases sociales rurales en particular para que, en vez de optar por impugnar el voto o anularlo, se hiciera efectivo para la fórmula contraria al gobierno de turno. En este sentido, el titular de la SRRC recordaba que:

\footnotetext{
“En esta provincia castigamos con nuestro voto a un gobernador soberbio y prepotente hace poco tiempo. En democracia los gobiernos no se derriban ni desestabilizan, se cambian. Este año tendremos otra vez la oportunidad de expresarnos en las urnas. Hágalo pensando en castigar la corrupción y la confiscación de su esfuerzo y el nuestro". ${ }^{43}$
}

Paradójicamente, se puede evidenciar en ese fragmento cómo, a pesar de que la crisis de representación implicaba un descentramiento de la política (Cavarozzi, 1996), es decir, una pérdida de la capacidad de los partidos políticos de intervenir efectivamente en la solución de los problemas de la ciudadanía, se continuaba considerando que la misma podía ejercer una intervención decisiva en la arena social y política. Paralelamente, también quedaba plasmado cómo el estilo de comunicación asumido por Mestre en su gestión fue un elemento que alimentó la conflictividad y que fue observable en el sentido político que asumieron las adjetivaciones (Riorda, 2004, p. 120) de los dirigentes rurales.

\section{A modo de síntesis}

El régimen social de acumulación que emergió de la crisis hiperinflacionaria de 1989 y que estuvo vigente hasta la salida de la convertibilidad cambiaria en enero de 2002 no solo alteró las estrategias de inversión y acumulación de los actores agrarios, sino que proyectó innovaciones en el campo de lo político. Por un lado, si la crítica coyuntura imponía una creciente convicción sobre la necesidad de un cambio de rumbo económico,

\footnotetext{
${ }^{41}$ AHMRC, H, $P$, domingo 15/09/1996, p. 4.

${ }^{42}$ AHMRC, H, $P$, lunes 22/02/1999, p. 9.

${ }^{43}$ AHMRC, H, $P$, miércoles 17/03/1999, p. 14. 
por el otro, la ideología neoliberal aparecía como el marco interpretativo (y operativo) a partir del cual debían encontrarse las soluciones.

Este contexto operó como el vector que le posibilitó a la SRRC canalizar representaciones sobre el Estado y la economía que eran previas (históricas). La seguridad jurídica, la inviolabilidad del derecho a la propiedad privada $y$, fundamentalmente, el tamaño sobredimensionado del Estado y su acción ineficiente que se manifestaban en un castigo al sector por medio de diferentes impuestos eran los tópicos centrales de la matriz discursiva liberal-conservadora que, en dicha coyuntura, reactualizaban su significación. Este proceso tuvo su contracara en los Estados provinciales, ámbito sobre el cual la SRRC y CARTEZ ejercían parte importante de su mediación política. El recambio del gabinete de gobierno en la provincia de Córdoba en 1995, aunque del mismo color político, se operaba en una situación de crisis que remitía a la que años antes se había dado a nivel nacional. Esta coyuntura, sumada a las exigencias del gobierno nacional, planteaba la necesidad de realizar severos ajustes en la administración provincial, afrontar la privatización de empresas estatales e incrementar los niveles de recaudación fiscal. En este proceso, las intervenciones públicas de la dirigencia agraria cordobesa apuntaban a manifestar su tradicional postura de que el Estado provincial racionalizara su accionar, es decir, que redujera su tamaño. En esa crítica se procuraba evadir la intención del gobierno provincial de apelar a incrementos de impuestos destinados al agro.

Dos cuestiones en torno a lo impositivo eran las que generaban mayores tensiones entre las entidades y el gabinete de Mestre. Por un lado, con respecto al impuesto inmobiliario, que constituía uno de los factores sobre los cuales históricamente se había dirigido su accionar, durante este período se inició un proceso de revalúo que incrementó sus valores. La dirigencia cuestionaba la progresividad del inmobiliario rural y lo hacía por medio de su prisma liberal-conservador: se consideraba una medida injusta de un Estado ineficiente. Por otro lado, en torno a las solicitudes de declaración de emergencia agropecuaria que implicaban la reducción o condonación de las cuotas del impuesto inmobiliario rural. En forma similar a lo anterior, se impusieron las acciones y críticas de la SRRC y de CARTEZ. Nuevamente en este aspecto se cuestionaban las maniobras dilatorias del gobierno provincial en cuanto a la declaración de emergencia. La demora en dar cabida a las demandas de los productores era considerada como un aspecto más de un Estado obsoleto que debía recurrir al excedente del agro para financiar sus déficits. A pesar de los contrapuntos que esta cuestión ocasionaba, la SRRC lograba ejercer una mediación política más o menos exitosa que se evidenciaba en la acogida por parte del gobierno provincial de las solicitudes de prórroga. Esto nos lleva a reconsiderar la cuestión de la marginalidad de las entidades agrarias en la relación con el Estado, al menos en la escala provincial. No obstante, es un aspecto que requiere profundizarse en próximas investigaciones. 
116 | "Alimentar las arcas del Estado": corporaciones agrarias, Estado y política...

\section{Referencias bibliográficas}

Arriaga, A. et al. (2012). Un Estado en transición: Córdoba ante el embate neoliberal. En M. Gordillo et al. La protesta frente a las reformas neoliberales en la Córdoba de fin de siglo (pp. 25-65). Córdoba: Ferreyra.

Ase, I. y Burijovich, J. (2001). La reinvención del estado cordobés: Una 'reingeniería' de la ciudadanía. Revista Administración Pública \& Sociedad, 14, 1-24.

Balsa, J. (2007). Las disputas hegemónicas en torno de las cuestiones sociales agrarias de la pampa argentina en la actualidad. En N. Girbal-Blacha y S. Mendonça (Coords.). Cuestiones agrarias en Argentina y Brasil (pp. 149-170). Buenos Aires: Prometeo.

Balsa, J. (2012). Formaciones discursivas y disputas por la hegemonía en torno a los modelos de desarrollo agrario. En S. Lázzaro y J. Balsa (Coords.). Agro y política en Argentina. El modelo agrario en cuestión, 1930-1943 (pp. 35-117). Buenos Aires: CICCUS.

Balsa, J. (2013). Los avatares de la reforma de la ley de colonización durante el primer peronismo (1946-1955). En N. Girbal-Blacha y S. Mendonça (Dirs.). Corporaciones agrarias y políticas públicas en América Latina (pp. 193-226). Rosario: Prohistoria.

Carini, G. (2011). Intereses agrarios y mediación corporativa en el sur de Córdoba. El discurso de la dirigencia agraria ante la nueva política peronista. En E. Escudero y R. Camaño Semprini (Comps.). Río Cuarto en tiempos del primer peronismo. Aproximaciones desde la Historia (pp.191-228). Córdoba: Ferreyra.

Carini, G. (2014). Reivindicaciones, discursos y conflicto: las corporaciones agrarias en el marco de la estructuración del 'consenso' neoliberal (1989-1991). Conflicto Social, 7 (12), 88-113.

Carini, G. (2014a). Reivindicaciones, discursos y estrategias en mutación: la Sociedad Rural de Río Cuarto en el marco de una 'nueva agricultura' (1990-2000). En G. Olivera et al. El agro cordobés en el siglo XX: entramados productivos, políticos y sociales desde una perspectiva histórica (pp. 223-280). Córdoba: Editorial de la Secretaría de Investigación, Ciencia y Técnica de la Facultad de Filosofía y Humanidades. Universidad Nacional de Córdoba.

Carini, G. (2015). Nuevo Estado, viejos intereses: corporaciones agrarias y mediación política en el interior de Córdoba (1935-1955). Estudios del ISHIR, 5 (11), 93-113.

Carini, G. (2017). Discursos, prácticas y estrategias en mutación: la Sociedad Rural de Río Cuarto ante el nuevo régimen social de acumulación de los noventa. Tesis de Doctorado en Historia. Facultad de Filosofía y Humanidades, Universidad Nacional de Córdoba [inédito].

Carini, G. (2017a). "Nuestro objetivo esencial es la defensa del sector que, en definitiva, son los intereses del país": asociaciones agrarias, lógicas institucionales y discursos en la pampa cordobesa. Estudios Rurales, 18 (13), 48-76. 
Carini, G. (2018). Agro, negocio y nueva institucionalidad en las pampas: itinerarios y propuestas de abordajes para el análisis de la representación de intereses agrarios. En G. Banzato et al. (Comps.). La Expansión de la frontera productiva y estructura agropecuaria. Buenos Aires: Prometeo-Asociación Argentina de Historia Económicas.

Carniglia, E. (2004). La mediatización de la ruralidad. Representaciones del espacio en la prensa especializada nacional. En G. Cimadevilla y E. Carniglia (Comps.). Comunicación, ruralidad y desarrollo. Mitos, paradigmas y dispositivos de cambio (pp. 285-306). Buenos Aires: INTA.

Carreras Doallo, X. (2012). Los medios gráficos como fuente. Un análisis de la revista La Chacra durante el peronismo clásico. Estudios Rurales, 1 (2), 184-201.

Carrizo, C. (2000). De la subordinación negativa a la subordinación positiva de la intervención estatal a los procesos de acumulación privada: el sistema político administrativo provincial en Córdoba, 1995- 1999. Revista Administración Pública y Sociedad, 13, pp. 1-9.

Cavarozzi, M. (1996). El capitalismo político tardío y su crisis en América Latina. Rosario: Homo Sapiens.

Cavarozzi, M. (1997). Autoritarismo y democracia (1955-1996). La transición del Estado al Mercado en la Argentina. Buenos Aires: Ariel.

Closa, G. (2010). La recuperación de la democracia y los gobiernos radicales. Angeloz y Mestre (1983-1999). En C. Tcach (Coord.). Córdoba Bicentenaria. Claves de su historia contemporánea (p. 463-493). Córdoba: Ferreyra.

Converso, F. (2008). El impuesto a la propiedad de la tierra. Córdoba 1914-1943. Revista de la Escuela de Historia de la UNSA, 7 (1), 1-40.

Fair, H. (2009). Una revisión crítica de los estudios sobre menemismo. Estudios, 8 (21), 105-129.

Gerchunoff, P. y Torre, J. C. (1996). La política de liberalización económica en la administración de Menem. Desarrollo Económico, 36 (143), 733-767.

Gordillo, M. (2010). Piquetes y cacerolas... El argentinazo de 2001. Buenos Aires: Sudamericana.

Gordillo, M. et al. (2012). La protesta frente a las reformas neoliberales en la Córdoba de fin de siglo. Córdoba: Ferreyra.

Gras, C. y Hernández, V. (2009). El fenómeno sojero en perspectiva: dimensiones productivas, sociales y simbólicas de la globalización agrorrural en la Argentina. En C. Gras y V. Hernández. La Argentina rural. De la agricultura familiar a los agronegocios (pp. 15-39). Biblos: Buenos Aires.

Gras, C. y Hernández, V. (2009). Los pilares del modelo agrobusiness y sus estilos empresariales. En C. Gras y V. Hernández (Coords.). El agro como negocio. Producción, sociedad y territorios en la globalización (pp. 17-49). Biblos: Buenos Aires.

La Serna, C. (2001). La Democracia como límite: el proceso de reforma del estado de la provincia de Córdoba en el Período 1995/1999. (pp. 1-32). En Actas del Primer Con- 
118 | "Alimentar las arcas del Estado": corporaciones agrarias, Estado y política...

greso de Administración Pública. Rosario: Asociación Argentina de Estudios de la Administración Pública.

La Serna, C. (2001). Reforma y democracia: el caso del Estado de la Provincia de Córdoba en el período 1995/1997. Revista Administración Pública y Sociedad, 14, 1-14.

Lardone, M. (2003). La reforma de los estados provinciales en la Argentina: hacia la construcción de un esquema analítico. Revista SAAP, 1 (2), 273-316.

Lattuada, M. (1992). Notas sobre corporaciones agropecuarias y Estado. Tendencias históricas y cursos de acción posibles en la experiencia democrática contemporánea. Estudios Sociales, 2 (1), 123-148.

Makler, C. (2007). Reflexiones sobre el gremialismo agropecuario en Argentina. En O. Graciano y S. Lázzaro (Comps.). La Argentina rural del siglo XX. Fuentes, problemas y métodos (pp. 343-371). Buenos Aires: La Colmena.

Martínez Nogueira, R. (1988). Las organizaciones del sector agropecuario. En O. Barsky et al. La agricultura pampeana. Transformaciones productivas y sociales (pp. 295-322). Buenos Aires: FCE, IICA, CISEA.

Olivera, G. (2008). Cooperativismo agrario: una nueva área temática en la renovación de la historia rural. En J. Balsa et al. (Comps.). Pasado y presente en el agro argentino (pp. 219-235). Buenos Aires: Lumiere.

Palermo, V. y Novaro, M. (1996). Política y poder en el gobierno de Menem. Buenos Aires: Norma.

Pasquino, G. (1996). “Participación política, grupos y movimientos”. En G. Pasquino et al. Manual de Ciencia Política (pp. 179-217). Madrid: Alianza.

Pucciarelli, A. (Coord.). Los años de Menem. La construcción del orden neoliberal. Buenos Aires: Siglo XXI.

Riorda, M. (2004). Mitos y política: estilos comunicativos de los gobernadores cordobeses (1983-2003). Estudios, 4 (15), 119-142.

Rosanvallon, P. (2003). Por una historia conceptual de lo político. Buenos Aires: Fondo de Cultura Económica.

Sanz Cerbino, G. (2014). Antecedentes históricos de la unidad de las corporaciones agropecuarias pampeanas. La formación de la Comisión de Enlace y la disputa por la renta (1966-1973). Mundo Agrario, 15 (29), 1-33.

Schmitter, P. (1991). “¿A dónde ha ido la teoría del neocorporativismo y hacia dónde puede ir su práctica?", en P. Schimitter et al. (Coords.). Neocorporativismo: más allá del Estado y el mercado. México: Alianza.

Schmitter, P. (1995). “Consolidación de la democracia y representación de grupos sociales". Cuadernos de Ciencias Sociales 76. Flacso: San José.

Scribano, A. (2003). Reflexiones sobre una estrategia metodológica para el análisis de las protestas sociales. Sociologías, 5 (9), 64-104.

Segura, M. (2007). Unión por Córdoba: La producción de una diferencia. En R. Costa y T. Mozejko (Comps.). Lugares del decir (pp. 249-282). Rosario: Homo Sapiens. 
Sidicaro, R. (2002). Los tres peronismos. Estado y poder económico 1946-55 / 1973-76 / 19891999. Buenos Aires: Siglo XXI.

Svampa, M. (2005). La sociedad excluyente. La Argentina bajo el signo del neoliberalismo. Buenos Aires: Taurus.

Tcach, C. (2006). Sabattinismo y peronismo. Partidos políticos en Córdoba (1943-1955). Buenos Aires: Biblos.

Thwaites Rey, M. (2002). La (des)ilusión privatista. El experimento neoliberal en la Argentina. Buenos Aires: Eudeba.

Para citar este artículo:

Carini, Gabriel Fernando (2018). "Alimentar las arcas del Estado": corporaciones agrarias, Estado y política en Córdoba (1995-1999). Anuario de la Escuela de Historia Virtual, 14, 100-119. 